\title{
Evolution of the Density of States Gap in a Disordered Superconductor
}

\author{
Carey Huscroft and Richard T. Scalettar \\ Department of Physics, University of California, Davis, CA 95616
}

\begin{abstract}
It has only recently been possible to study the superconducting state in the attractive Hubbard Hamiltonian via a direct observation of the formation of a gap in the density of states $N(\omega)$. Here we determine the effect of random chemical potentials on $N(\omega)$ and show that at weak coupling, disorder closes the gap concurrently with the destruction of superconductivity. At larger, but still intermediate coupling, a pseudogap in $N(\omega)$ remains even well beyond the point at which off-diagonal long range order vanishes. This change in the elementary excitations of the insulating phase corresponds to a crossover between Fermiand Bose-Insulators. These calculations represent the first computation of the density of states in a finite dimensional disordered fermion model via the Quantum Monte Carlo and maximum entropy methods.
\end{abstract}

PACS numbers: 74.20.Mn 74.30.+h 74.20.-z 71.55.Jv

(March 6, 2018)

Analysis of the effect of randomness on the superconducting state has a long history, [1] beginning with Anderson's observation [2] that in the presence of nonmagnetic disorder the Cooper wavefunction can be constructed by replacing $(k \uparrow,-k \downarrow)$ pairs by an appropriate combination of time reversed, but still extended, eigenstates. Recent theoretical work [3] has attempted to understand experiments [4] on the superconductinginsulator (SC-I) transition in thin films. Here, the superconducting phase is destroyed by strong disorder which localizes the electrons entirely. Numerical work [5] has focussed on boson models in which coherence of the pair phase is the central issue, and the role of fluctuations in the pair amplitude is suppressed. Granular systems might be well described by such pre-formed Cooper pairs, and, furthermore, scaling arguments suggest universal conductivity might find a natural explanation within boson systems. [3]

Despite the successes of numeric studies of interacting, disordered bose systems, it is clear that explicit calculations for itinerant electron models, in which pairs can break apart, are essential. Experimentally, there is evidence for both SC-I transitions to a Bosonic state [6] and to a Fermionic state [7], distinguished by the existence or lack of an energy gap, respectively, in the insulating phase. There is currently considerable controversy regarding the meaning of the two insulting phases seen experimentally. [8] Theories implicating both Bosons [9] and Fermions $10 \|$ exist.

In this paper we describe the evolution of the singleparticle density of states for the disordered, attractive Hubbard Hamiltonian, a model which can interpolate between Bose and Fermi limits. We show that increasing disorder destroys long range pairing correlations and drives a superconducting-insulator phase transition. However, the density of states $N(\omega)$ shows a gap which closes with the destruction of superconducting long-range order for relatively weak couplings yet retains a gap be- yond the critical disorder strength for larger couplings. Sweeps across the disorder-interaction phase diagram allow us to locate quantitatively the critical coupling strengths for gap and pair order formation in a set of representative cases.

The attractive Hubbard Hamiltonian, in the presence of random site energies is,

$$
\begin{aligned}
H= & -t \sum_{\langle\mathbf{i} \mathbf{j}\rangle \sigma}\left(c_{\mathbf{i} \sigma}^{\dagger} c_{\mathbf{j} \sigma}+c_{\mathbf{j} \sigma}^{\dagger} c_{\mathbf{i} \sigma}\right)-\sum_{\mathbf{i} \sigma}\left(\mu-v_{\mathbf{i}}\right)\left(n_{\mathbf{i} \sigma}-\frac{1}{2}\right) \\
& -|U| \sum_{\mathbf{i}}\left(n_{\mathbf{i} \uparrow}-\frac{1}{2}\right)\left(n_{\mathbf{i} \downarrow}-\frac{1}{2}\right) .
\end{aligned}
$$

Here $c_{\mathbf{i} \sigma}$ is a fermion destruction operator at site $\mathbf{i}$ with $\operatorname{spin} \sigma, n_{\mathbf{i} \sigma}=c_{\mathbf{i} \sigma}^{\dagger} c_{\mathbf{i} \sigma}$, and the chemical potential $\mu$ fixes the average density $\langle n\rangle$. The site energies $v_{\mathbf{i}}$ are independent random variables with a uniform distribution over $[-V / 2, V / 2]$. The interaction has been written in particle-hole symmetric form so that $\mu=0$ corresponds to $\langle n\rangle=1$ at all $U$ and $T$ when $V=0$. The lattice sum $\langle\mathbf{i j}\rangle$ is over near neighbor sites on a two dimensional square lattice.

We solve for the equilibrium properties of this Hamiltonian using the determinant QMC method. 11] Previous numerical studies of the clean model have determined the phase diagram [12 by a finite size scaling analysis of the equal time pair-pair correlation functions,

$$
P_{s}(\mathbf{j})=\frac{1}{N} \sum_{\mathbf{i}}\left\langle\Delta_{\mathbf{i}+\mathbf{j}} \Delta_{\mathbf{i}}^{\dagger}\right\rangle, \quad \Delta_{\mathbf{j}}^{\dagger}=c_{\uparrow \mathbf{j}}^{\dagger} c_{\downarrow \mathbf{j}}^{\dagger}
$$

The phase diagram consists of a state with simultaneous charge density wave (CDW) and superconducting correlations at $T=0$ and half-filling, and a finite temperature Kosterlitz-Thouless transition (with a maximal $T_{c} \approx 0.1 t$ for $|U|=4 t$ ) to a phase with power law decay of pairing correlations off half-filling. In this paper we will study fillings $\langle n\rangle=0.875$ for which strong CDW 
correlations are absent and the transition temperature to the SC phase is nearly maximal.

In Fig. 1 we show the suppression of long range correlations in $P_{s}(\mathbf{j})$ with increasing disorder strength. A finite size scaling analysis of the structure factor determines the critical value $V_{c}=(3.25 \pm 0.2) t$ for the destruction of the superconducting state. $V_{c}$ can also be identified by the superfluid density $D_{s}$ and the conductivity. [13] These quantities give consistent values, $V_{c}=(3.2 \pm 0.7) t$ and $V_{c}=(3.5 \pm 0.5) t$, respectively, for the critical disorder.

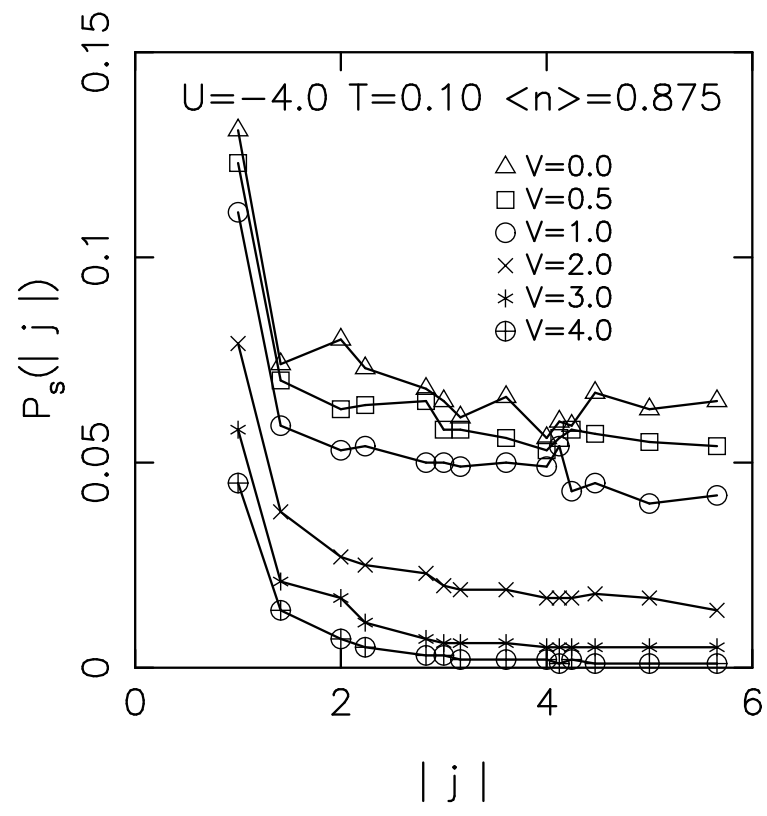

FIG. 1. The equal time pair correlation $P_{s}(\mathbf{j})$ is shown at $T=0.10$ as a function of separation $\mathbf{j}$ for different degrees of disorder. $P_{s}(\mathbf{j})$ remains finite at large $\mathbf{j}$ for $V=2.0$, but goes to zero rapidly with $\mathbf{j}$ at $V=4.0$.

The density of states is a quantity which, like the conductivity, is directly accessible experimentally. Its evaluation requires an inversion of the integral relation,

$$
G(\tau)=\frac{1}{N} \sum_{\mathbf{p}}\left\langle c_{\mathbf{p}}(\tau) c_{\mathbf{p}}^{\dagger}(0)\right\rangle=\int_{-\infty}^{+\infty} d \omega \frac{e^{-\omega \tau} N(\omega)}{1+e^{-\beta \omega}}
$$

between the density of states $N(\omega)$ and the imaginary time dependent Greens function $G(\tau)$. We do this using Bryan's method [14] and Classic Maximum Entropy [15], using the full imaginary-time covariance matrix.

Although this approach is fairly well-developed for clean systems, the inclusion of disorder raises a number of new questions of principle. The proper treatment of errors and correlations in the QMC data was the central achievement of the ME approach, [16] and it is not obvious how additional fluctuations from disorder averaging will affect the procedure. Therefore, we have checked our calculation against analytic results both at weak and strong coupling. Besides verifying the numerics, the be- havior in these two limits also presages the two distinct types of insulating behavior we see in the full model.

In the non-interacting limit we can diagonalize the Hamiltonian and obtain $E_{\alpha}$, the single particle eigenenergies and thence $N(\omega)=\frac{1}{N} \sum_{\alpha} \delta\left(\omega-E_{\alpha}\right)$. In this limit the determinant QMC method computes the imaginary time fermion Greens function exactly. Tests of the ME approach in the clean case require that some noise be added to the exact $G(\tau)$ coming from the QMC. [17] In the random case, this ad hoc noise does not need to be added, since $G(\tau)$ already has error bars from disorder averaging. We find that the ME approach exactly tracks the disorder induced broadening of $N(\omega)$. 18 For small $|U|$ we expect disorder to broaden and eliminate the superconducting gap as well.

The strong coupling, $t=0$, limit also can be solved analytically. For a single site,

$$
\begin{aligned}
G(\tau) & =\frac{e^{-\beta U / 4} e^{-\tau \Delta_{-}}+e^{\beta\left(U / 4+v_{\mathbf{i}}\right)} e^{-\tau \Delta_{+}}}{2\left(e^{\beta U / 4} \cosh \beta v_{\mathbf{i}}+e^{-\beta U / 4}\right)}, \\
N(\omega) & =\frac{\left(1+e^{-\beta \Delta_{-}}\right) e^{-\beta U / 4}}{2\left(e^{\beta U / 4} \cosh \beta v_{\mathbf{i}}+e^{-\beta U / 4}\right)} \delta\left(\omega-\Delta_{-}\right) \\
& +\frac{\left(1+e^{-\beta \Delta_{+}}\right) e^{\beta\left(U / 4+v_{\mathbf{i}}\right)}}{2\left(e^{\beta U / 4} \cosh \beta v_{\mathbf{i}}+e^{-\beta U / 4}\right)} \delta\left(\omega-\Delta_{+}\right) .
\end{aligned}
$$

Here $\Delta_{ \pm}= \pm U / 2+v_{\mathbf{i}}$. After disorder averaging, the two delta functions in the density of states $N(\omega)$ are broadened to two distributions centered about $\pm U / 2$, each with width $V$. When $V=U$ these merge, and the gap in $N(\omega)$ at $\omega=0$ is closed. However, the thermal factors greatly suppress $N(\omega)$ near $\omega=0$. At $t=0$ there is no long range pairing order, so that this "pseudo-gap" in the density of states reflects the tendency for on-site singlet formation. We have shown that our QMC+ME procedure accurately reproduces the analytic result Eq. 4 as a function of temperature, disorder strength, and interaction strength. 18] A central conclusion of our work is that this pseudogap behavior persists far from the strong-coupling limit as the hopping $t$ is turned on.

We now study the cross--over between these two possible types of effect of disorder on $N(\omega)$ in the full model. In Fig. 2 we show the evolution of $N(\omega)$ with increasing interaction strength $|U|$ at fixed disorder $V=2 t . N(\omega)$ evolves from its gapless, noninteracting form to possessing a well-formed gap at $|U|=4 t$. Similarly, in Fig. 3 we show the evolution of $N(\omega)$ with increasing disorder $V$, at fixed values of the interaction, $|U|=4 t$. In contrast to the non-interacting case where increasing $V$ results in the expected broadening of $N(\omega)$, here the gap in $N(\omega)$ is remarkably robust. Note the values of $V$ are well beyond the point where the superconducting-insulator transition has occurred, as indicated by the results in Fig. 1 and related measurements. [13 In the repulsive Hubbard model at the same value of $U / t$ the gap in $N(\omega)$ requires long range antiferromagnetic order be present, specifically, the 
correlation length $\xi_{\text {af }}$ must exceed the lattice size. 19] In contrast, here we find that a gap in $N(\omega)$ persists despite the clear destruction of such long range order.

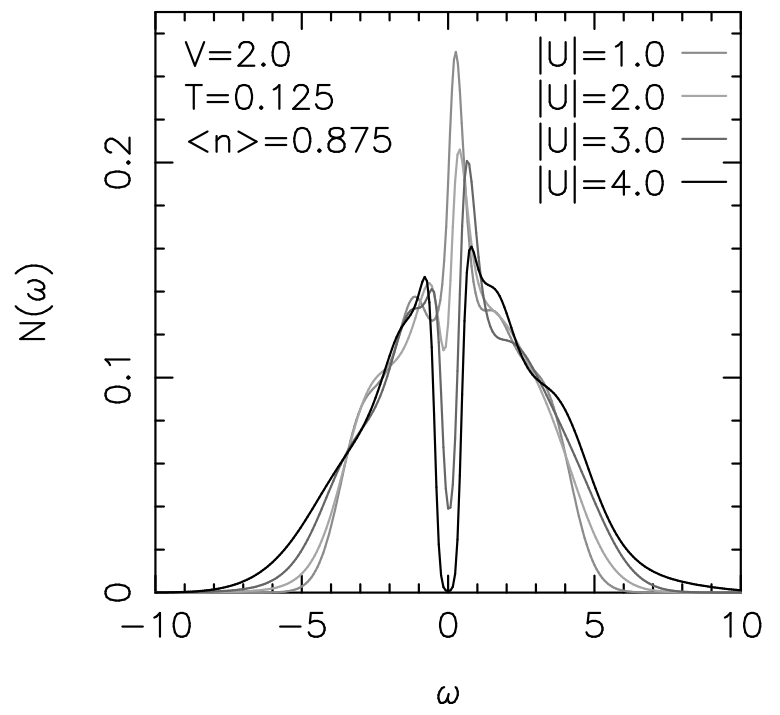

FIG. 2. The density of states $N(\omega)$ at $t=1, T=0.125$, $V=2,|U|=1,2,3,4$ on an $8 \times 8$ lattice. For sufficiently small $|U|$, scattering by the disorder inhibits phase coherence and the superconducting gap is destroyed. A superconducting gap forms with increasing interaction strength $U$. A study of the pair correlations $P_{s}(\mathbf{j})$ indicates that gap formation occurs precisely with the onset of superconductivity.

How can we interpret these results? In the weakcoupling limit, disorder broadens $N(\omega)$ at all energies. In particular, the superconducting gap in $N(\omega)$ is closed at the same point where disorder destroys the long-range pair correlations. This closing of the gap is indicative of the predominance of fermionic elementary excitations in the insulating phase. In the strong coupling limit, the density of states is not broadened by disorder near $\omega=0$ and a pseudogap survives. The results of Fig. 3 imply that already at a value of interaction strength $|U|=4 t$ which is only one-half the bandwidth, $W=8 t$, the superconducting gap is robust to disorder. In previous work, [20] it was found that at $V=0$ and $|U|=4 t$ the spin susceptibility exhibits a reduction at low temperatures, indicative of bose-like behavior, despite the fact that the temperature dependence of the chemical potential $\mu(T)$ is still clearly that of a degenerate Fermi system. We conclude here that for an interaction strength as low as $|U|=4 t$, the density of states reflects bose-like character at all $V$. As shown in Fig. 3, at either side of the critical disorder $V_{c}$ for the destruction of long-range order in this coupling regime, there are no indications of fermionic excitations in $N(\omega)$.

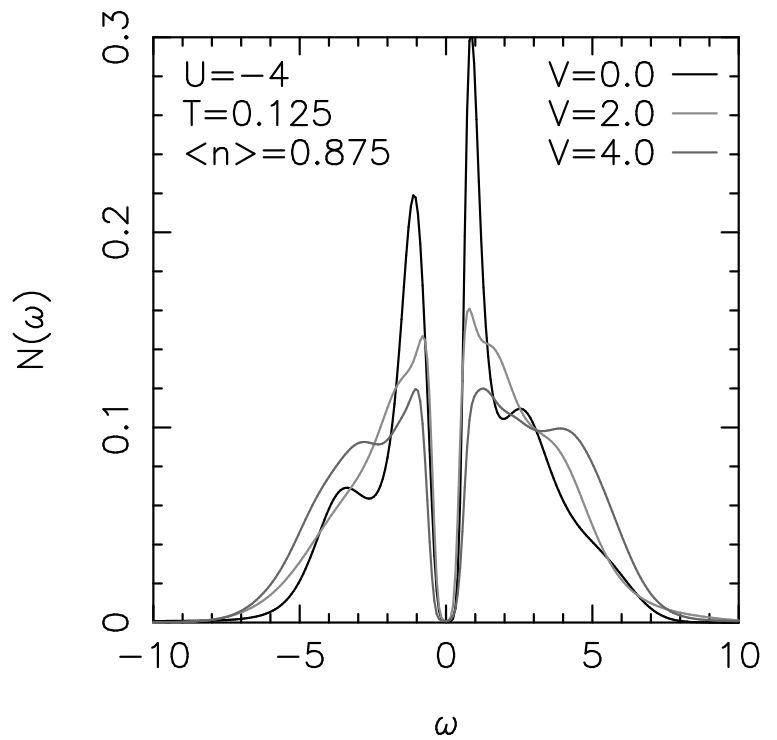

FIG. 3. The density of states $N(\omega)$ at $t=1, U=-4$, $T=0.125, V=0.0,2.0,4.0$ on an $8 \times 8$ lattice. A study of the equal time pair correlation shows that for $V<V_{c} \approx 3.5$ the system is a superconductor. For $V \geq V_{c}$ the system is an insulator. The gap in $N(\omega)$ persists even after long-range pair correlations, and superconductivity, are destroyed. Furthermore, there is no enhancement in $N(\omega)$ as one approaches $V_{c}$ from either side, indicating that the system is not a Fermi liquid at the $\mathrm{SC}-\mathrm{BI}$ transition.

The phase diagram is shown in Fig. 4. For weak interactions, there is a SC-Fermi Insulator (SC-FI) transition with disorder as seen in one class of experiments. [7] For a stronger, but nevertheless intermediate coupling, the phase diagram already exhibits the SC-Bose Insulator (SC-BI) transition seen in another class of experiments. [6] From Eq. 4 it follows that for small $t$ the pseudogap in $N(\omega)$ opens even for very small $|U| / V$ at large $\beta$, indicating that the FI-BI crossover has the trajectory shown. [18] Theoretically, a universal resistivity is predicted by a bosonic treatment of the transition. [9] It has been suggested that the presence of fermions causes non-universal properties at the SC-I transition. 21] It is possible that the attractive Hubbard Model will exhibit universal resistivity only in the Bose-Insulator regime, though considerable further work needs to be done to settle this issue.

The interplay of finite-size effects and the development of a gap in $N(\omega)$ is a subtle issue. As described above, in the case of the half-filled repulsive Hubbard model at $U=4 t$, an antiferromagnetic gap in $N(\omega)$ at finite temperature disappears when the lattice size is increased beyond the correlation length of antiferromagnetic order. We have studied this issue carefully by further evaluation of $N(\omega)$ on a range of lattices, and conclude that the gap we observe is a robust feature and not an artifact of finite lattice size. A crucial difference here from the case of the half-filled repulsive model is that the antiferromagnetic 
transition takes place only at $T=0$, whereas the superconducting transition off half-filling in the attractive model takes place at finite temperature.

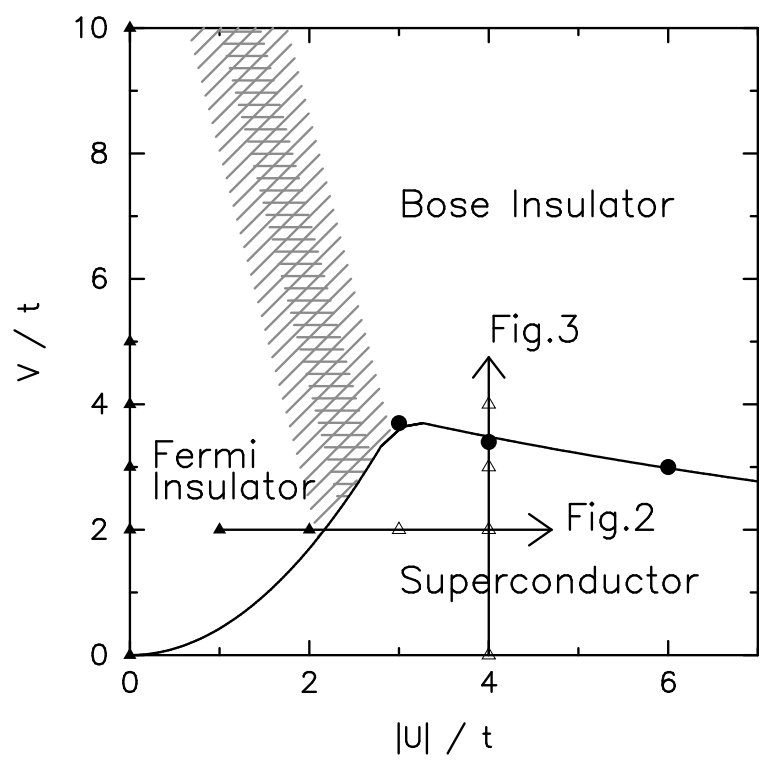

FIG. 4. Phase diagram as a function of disorder $V / t$ and interaction strength $|U| / t$. The solid circles indicate the location of the entry into the superconducting phase as determined by our simulations. The remainder of the solid line indicates an estimate of the location of the boundary of the superconducting region. The arrows correspond to Figs. 2 and 3, open triangles indicate gapped regions while closed triangles indicate no gap in $N(\omega)$. In the superconducting regime the pair correlations have long range order and there is a gap in $N(\omega)$. In the insulating phases there is no pair long range order. The hatched region demarks schematically the cross-over between the Bose and Fermi Insulators.

In conclusion, we have computed the density of states $N(\omega)$ in a disordered interacting fermion model with a combination of the QMC and ME methods. The disorder-induced broadening expected in the weakcoupling BCS limit is already absent by the time $|U|=4 t$, half the single particle bandwidth $W=8 t$. Disorder closes the gap in the density of states for weak interactions but for stronger interactions has little effect on the gap well beyond the point where pair coherence vanishes and the system is insulating. At the coupling where a metallic state has been previously identified, the density of states suggests bosonic excitations on either side of the $\mathrm{SC}-\mathrm{I}$ transition.

We gratefully acknowledge support from the SDSC and the NSF under grant No. DMR-9528535, as well as useful discussions with N. Trivedi and M. Ulmke.

[1] P. A. Lee and T. V. Ramakrishnan, Rev. Mod. Phys. 57, 287 (1985); D. Belitz and T. Kirkpatrick, Rev. Mod. Phys. 66, 261 (1994).
[2] P.W. Anderson, Phys. Rev. 109, 1492 (1958).

[3] M.P.A. Fisher, et al. Phys. Rev. B40, 546 (1989).

[4] D.B. Haviland, Y. Liu, and A.M. Goldman, Phys. Rev. Lett. 62 2180, (1989); A.F. Hebard and M.A. Paalanen, Phys. Rev. Lett. 65, 927 (1990); and J.M. Valles, R.C. Dynes, and J.P. Garno, Phys. Rev. Lett. 69, 3567 (1992); A. Yazdani and A. Kapitulnik, Phys. Rev. Lett. 74, 3037 (1995).

[5] M.C. Cha, M.P.A. Fisher, S.M. Girvin, M. Wallin, and A.P. Young, Phys. Rev. B44, 6883 (1991); R. T. Scalettar, G. G. Batrouni and G. T. Zimanyi, Phys. Rev. Lett. 66, 3144 (1991). W. Krauth, N. Trivedi and D. M. Ceperley, Phys. Rev. Lett. 67, 2307 (1991); M. Makivic, N. Trivedi and S. Ullah, Phys. Rev. Lett. 71, 2307 (1993); M. Wallin, E.S. Sorensen, S.M. Girvin and A.P. Young, Phys. Rev. B49, 12115 (1994).

[6] M.A. Paalanen, A.F. Hebard, and R.R. Ruel, Phys. Rev. Lett. 69, 1604 (1992); A.F. Hebard, Strongly Correlated Electronic Materials, eds. K.S. Bedell, et al. p. 251 (1993).

[7] J.M. Valles, Jr., S. Hsu, R.C. Dynes, and J.P. Garno, Physica B197, 522 (1994).

[8] D. Das and S. Doniach, unpublished, (cond-mat 9803345).

[9] M.P.A. Fisher, G. Grinstein, and S.M. Girvin, Phys. Rev. Lett. 64, 587 (1990).

[10] D. Belitz, Phys. Rev. B40, 111 (1989); S. Maekawa and H. Fukuyama, J. Phys. Soc. Jap. 51, 1380 (1982); K. Wagenblast, A. van Otterlo, G. Schön, and G.T. Zimányi, Phys. Rev. Lett. 79, 2730 (1997).

[11] R. Blankenbecler, R.L. Sugar, and D.J. Scalapino, Phys. Rev. D24, 2278 (1981); S. R. White, et al. Phys. Rev. B40, 506 (1989).

[12] R.T. Scalettar et al. Phys. Rev. Lett. 62, 1407 (1989); A. Moreo, D.J. Scalapino, and S.R. White, Phys. Rev. B45, 7544 (1992). F.F. Assaad, R. Preuss, A. Muramatsu, and W. Hanke, J. Low Temp. Phys. 95, 251 (1994).

[13] N. Trivedi, R.T. Scalettar, and M. Randeria, Phys. Rev. B54, 3756 (1996).

[14] R.K. Bryan, Eur. Biophys. J. 18, 165 (1990).

[15] M. Jarrell and J.E. Gubernatis, Phys. Rep. 269, 135 (1996).

[16] This treatment of errors and qualifying both the data and the results of a ME analytic continuation in the nondisordered case are discussed at length in Ref. [15].

[17] S.R. White, Phys. Rev. B44, 4670 (1991). At stronger couplings, $U=8 t$, a gap in $N(\omega)$ does exist even with only short range magnetic order.

[18] C. Huscroft and R.T. Scalettar, unpublished.

[19] S.R. White, Phys. Rev. B46, 5678 (1992).

[20] M. Randeria, N. Trivedi, A. Moreo, and R.T. Scalettar, Phys. Rev. Lett. 69, 2001 (1992).

[21] A. Yazdani and A. Kapitulnik, Phys. Rev. Lett. 74, 3037 (1995). 